\title{
O-GIcNAcylation is increased in prostate cancer tissues and enhances malignancy of prostate cancer cells
}

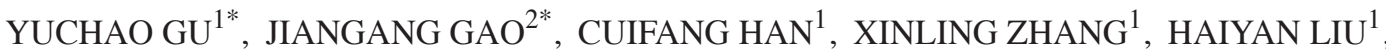 \\ LEINA MA ${ }^{1}$, XIAOQING SUN ${ }^{2}$ and WENGONG YU ${ }^{1}$ \\ ${ }^{1}$ Key Laboratory of Marine Drugs, Chinese Ministry of Education, \\ Key Laboratory of Glycoscience and Glycotechnology of Shandong Province, \\ School of Medicine and Pharmacy, Ocean University of China, Qingdao 266003; \\ ${ }^{2}$ Department of Urology Surgery, Qingdao Municipal Hospital, Qingdao 266011, P.R. China
}

Received November 7, 2013; Accepted March 14, 2014

DOI: $10.3892 / \mathrm{mmr} .2014 .2269$

\begin{abstract}
O-GlcNAc is an O-linked $\beta$-N-acetylglucosamine moiety attached to the side-chain hydroxyl of a serine or threonine residue in numerous cytoplasmic and nuclear proteins. In this study, we detected the level of O-GlcNAc in prostate, liver and pancreatic cancer tissues, and found that the global O-GlcNAc modification also known as O-GlcNAcylation, is specifically increased in prostate cancer tissues compared to corresponding adjacent tissues. In addition, we found that global O-GlcNAcylation is increased in prostate cancer cells and not in benign prostatic hyperplasia (BPH) epithelial cells. $\mathrm{O}-\mathrm{GlcNAc}$ enhanced the anchorage-independent growth and the migratory/invasive ability of prostate cancer cells. More importantly, we provide here, for the first time to the best of our knowledge, direct evidence that increased O-GlcNAcylation induces malignant transformation of nontumorigenic (BPH) cells. Furthermore, our study suggested that inhibiting the formation of the E-cadherin/catenin/cytoskeleton complex may underly the O-GlcNAc-induced prostate cancer progression. Overall, these findings indicated that O-GlcNAcylation is increased in prostate, but not in liver and pancreatic cancer tissues, and that O-GlcNAc can enhance the malignancy of prostate cancer cells.
\end{abstract}

\section{Introduction}

Numerous nuclear and cytoplasmic proteins have been found modified with $\mathrm{O}-\beta-\mathrm{N}$-acetylglucosamine $(\mathrm{O}-\mathrm{GlcNAc})$ at the

Correspondence to: Professor Xiaoqing Sun, Department of Urology Surgery, Qingdao Municipal Hospital, 5 Donghai Zhong Road, Qingdao 266011, P.R. China

E-mail: sunny466@163.com

*Contributed equally

Key words: O-GlcNAc, prostate cancer, liver cancer, pancreatic cancer, E-cadherin hydroxyl moiety of serine or threonine residues. This moiety is dynamically added and removed by the O-GlcNAc transferase (OGT) and the O-GlcNAcase (OGA) enzymes, respectively (1). UDP-GlcNAc is the donor substrate of OGT, and is biosynthesized through the hexosamine pathway (HBP). The HBP flux is highly dependent on glucose and glutamine, with $\sim 3-5 \%$ of total glucose entering this pathway (2). O-GlcNAc is a sensor of intracellular glucose metabolism, since the intracellular level of UDP-GlcNAc is the main regulatory factor for the activity of OGT (3). O-GlcNAcylation is altered in metabolism-associated diseases, such as type II diabetes (4-6), Alzheimer's disease (7) and cancer (8). Therefore, abnormal O-GlcNAcylation may play critical roles in these pathological processes.

Aberrant metabolism is a hallmark of cancer. Most cancer cells show increased rates of glucose and glutamine utilization, up to 200 -fold higher than those observed in the healthy cells they originate from, and predominantly produce energy through glycolysis followed by lactic acid fermentation $(9,10)$. As a glycolytic pathway, HBP can enhance the level of UDP-GlcNAc, and thus, the activity of OGT, which may lead to an increase in the global O-GlcNAc level (summed over all proteins) in cancer cells. We have previously demonstrated that the global O-GlcNAc level is increased in breast, lung and colon cancer tissues as compared to respective adjacent tissues $(11,12)$. In addition, other research groups have shown that O-GlcNAcylation is increased in breast, prostate and pancreatic cancer cell lines (13-15). However, whether increased O-GlcNAcylation is universal in cancer is unknown.

In this study, we examined the O-GlcNAc level in prostate, pancreatic and liver cancer tissues, and the results indicated that global O-GlcNAcylation is specifically increased in prostate cancer tissues compared to corresponding adjacent tissues. Furthermore, we investigated the roles of O-GlcNAc in prostate cancer cell proliferation and invasion, along with the underlying mechanism.

\section{Materials and methods}

Specimens and ethics. Liver, pancreatic and prostate cancer tissue microarrays (TMAs; OD-CT-DgLiv03-002, 
OD-CT-DgPan03-002, and OD-CT-UrPrt03-001 respectively) were purchased from Shanghai Outdo Biotech Co. (Shanghai, China). The liver cancer tissue TMA was constructed with 31 formalin-fixed, paraffin-embedded hepatocellular carcinoma tissues and their corresponding adjacent liver tissues, the pancreatic cancer tissue TMA was constructed with 31 formalin-fixed, paraffin-embedded pancreatic ductal adenocarcinoma tissues and their corresponding adjacent pancreatic tissues, and the prostate cancer tissue TMA was constructed with 29 formalin-fixed, paraffin-embedded prostate cancer tissues and their corresponding adjacent benign prostatic hyperplasia (BPH) tissues. In addition, 26 prostate cancer tissues and $19 \mathrm{BPH}$ tissues, which were fixed with formalin and embedded with paraffin, were obtained from the Department of Urology Surgery, Qingdao Municipal Hospital (QMH). The tissues were cut into $6-\mu \mathrm{m}$-thick sections and mounted on glass slides. Written informed consent was acquired from all patients and/or guardians for the use of their tissue samples. The present study was reviewed and approved by the Research Ethics Committee of Qingdao Municipal Hospital (Qingdao, China).

Immunohistochemistry. Immunohistochemistry was performed on the specimens using the DAKO Liquid DAB Substrate Chromogen system (Dako North America, Inc., Carpinteria, CA, USA) and a monoclonal mouse antibody targeting O-GlcNAc (RL2; Affinity BioReagents, Inc., Golden, CO, USA), used at a 1:200 dilution. The Fromowitz standard was used to semi-quantitatively assess the staining intensity of O-GlcNAc $(16,17)$.

Cell cultures. The human BPH-1 line is representative of benign prostatic hyperplasia, while the PC3 and DU145 prostate cancer cell lines are representative of the earlier type-I ADI prostate cancers. All cell lines were maintained in RPMI-1640 medium (Gibco, Grand Island, NY, USA) supplemented with $10 \%$ fetal bovine serum (FBS; Corning Cellgro, Manassas, VA, USA). To increase the O-GlcNAc level, cells were treated for $24 \mathrm{~h}$ or the indicated time period with $10 \mu \mathrm{M}$ thiamet-G, a selective OGA inhibitor, synthesized as previously described (18).

Immunoblotting (IB). Cells were lysed in lysis buffer [50 mM Tris- $\mathrm{HCl}$ (pH 7.4), $150 \mathrm{mM} \mathrm{NaCl}, 1 \%$ NP40, 1 mM EDTA, $1 \mathrm{mM} \mathrm{Na} \mathrm{VO}_{4}, 10 \mathrm{mM} \mathrm{NaF}$ ] containing a protease inhibitor cocktail (Roche Applied Science, Mannheim, Germany), $50 \mathrm{mM}$ GlcNAc and $5 \mu \mathrm{M}$ PUGNAc (both from Toronto Research Chemicals Inc., North York, ON, Canada). Protein samples $(30 \mu \mathrm{g})$ were separated by $7.5 \%$ dodecyl sulfate (SDS)-polyacrylamide gel electrophoresis (PAGE) and transferred to Immobilon-P membranes (Millipore, Billerica, MA, USA). Antibodies targeting O-GlcNAc (CTD110.6; Abcam, Cambridge, UK), glyceraldehyde 3-phosphate dehydrogenase (GAPDH), OGT (F-12) and OGA (all from Santa Cruz Biotechnology, Inc., Santa Cruz, CA, USA), were used along with the Amersham ECL Prime (GE Healthcare, Buckinghamshire, UK).

Soft agar assay. The soft agar assay was performed as described in (19). Briefly, cells $\left(1 \times 10^{4}\right)$ were suspended in $1 \mathrm{ml}$ top agar medium (RPMI-1640 medium supplemented with $10 \%$ FBS in $0.4 \%$ agar). The cell suspension was then overlaid onto $1.5 \mathrm{ml}$ bottom agar medium (the corresponding medium in $0.8 \%$ agar) in 6-well tissue culture plates in triplicate. Fresh medium was added to the plates every 3 days as a feeder layer. On the 5 th day, images were acquired from 6 random fields of the colonies at X40 magnification.

Cell invasion and migration assay. The cell invasion and migration assays were performed as described in (19). Transwell chambers (6.5 mm; Corning, New York, NY, USA) with $8 \mu \mathrm{m}$-pore membranes were used for the cell migration assay. The lower chamber was filled with RPMI-1640 medium (supplemented with 20\% FBS) and thiamet-G, except for control samples. Cells $\left(5 \times 10^{4}\right)$ were suspended in $100 \mu$ upper medium (RPMI-1640 medium with 1\% FBS) and placed into the upper chamber with or without thiamet-G. After $16 \mathrm{~h}$, the number of cells detected by crystal violet staining on the undersurface of the polycarbonate membranes was visually scored in five random fields at x100 magnification using a light microscope.

The invasion assay was performed using the same procedure as the migration assay, except that the upper surface of the membrane was covered with $70 \mu 1$ of $1 \mathrm{mg} / \mathrm{ml}$ Matrigel (BD Biosciences, San José, CA, USA) and the incubation time was prolonged to $24 \mathrm{~h}$.

RNA isolation and quantitative reverse transcription (RT)-PCR. Total RNA was isolated from the cells using an RNA isolation kit (BioTeke Corporation, Beijing, China), and reverse transcription was carried out using the M-MLV reverse transcriptase (Takara Biotechnology Co., Ltd., Dalian, China). Quantitative PCR assays on the resulting cDNA products were carried out in triplicate using the SYBR ${ }^{\circledR}$ Green PCR Master mix (Applied Biosystems/Life Technologies, Carlsbad, CA, USA) on a 7500 Real-time PCR system (Applied Biosystems/Life Technologies). The levels of the tested genes were normalized to that of the endogenous control gene GAPDH. The primers for amplification used were the following: human E-cadherin forward, 5'-GCC ACC CTG GCT TTG ACG-3', and reverse, 5'-CCA TCT GTG CCC ACT TTG AAT C-3'; human GAPDH forward, 5'-CAG GGC TGC TTT TAA CTC TGG T-3', and reverse 5'-CCT GGA AGA TGG TGA TGG GAT-3'.

Extraction of cytoskeleton-binding proteins. To separate cytoskeleton-binding proteins from other cell constituents, BPH-1 and DU145 cells were washed twice with phosphate-buffered saline, lysed on ice for $15 \mathrm{~min}$ in Triton X-100 lysis buffer [300 mM sucrose, 10 mM PIPES (pH 6.8), 50 mM NaCl, 3 mM $\mathrm{MgCl}_{2}, 0.5 \%$ Triton X-100, $0.1 \mathrm{mg} / \mathrm{ml}$ DNase, $0.1 \mathrm{mg} / \mathrm{ml}$ RNase, $1.2 \mathrm{mM}$ phenylmethanesulfonyl fluoride and protease inhibitor mix cocktail], passed through a 26 gauge needle four times, then subjected to centrifugation at $48,000 \mathrm{x}$ g for $10 \mathrm{~min}$ at $4^{\circ} \mathrm{C}$. The pellet was solubilized in loading buffer containing $2 \%$ SDS, and the supernatant and pellet fractions were loaded onto separate gels and processed as previously described (20).

Statistical analysis. Data were analyzed by Student's t-tests using the SPSS 11.0 software (IBM, Armonk, NY, USA). P<0.05 was considered to indicate statistically significant differences. Data were expressed as the mean \pm SD. 
A

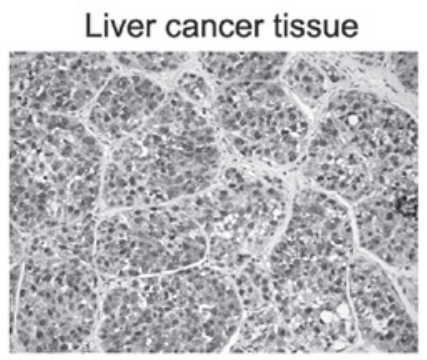

B
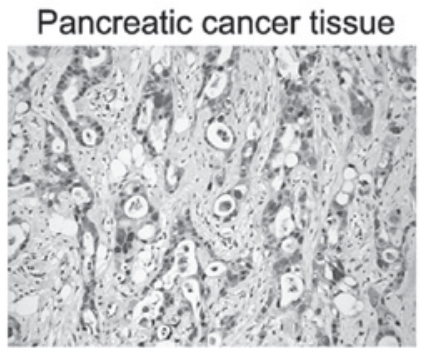

C

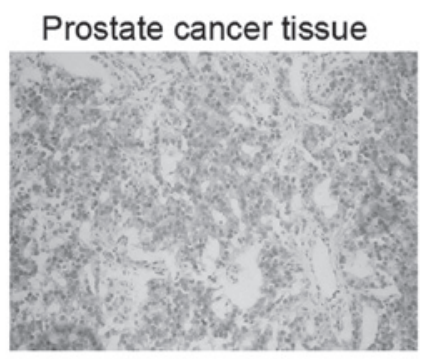

Adjacent tissue

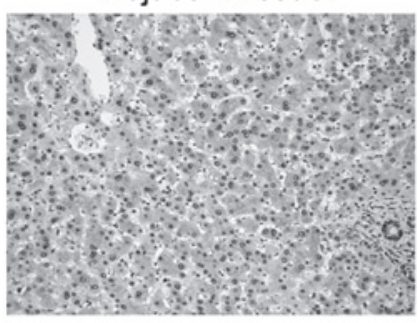

Adjacent tissue
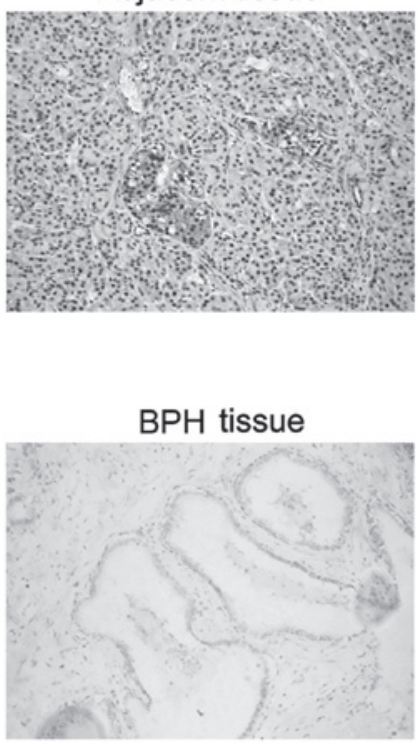
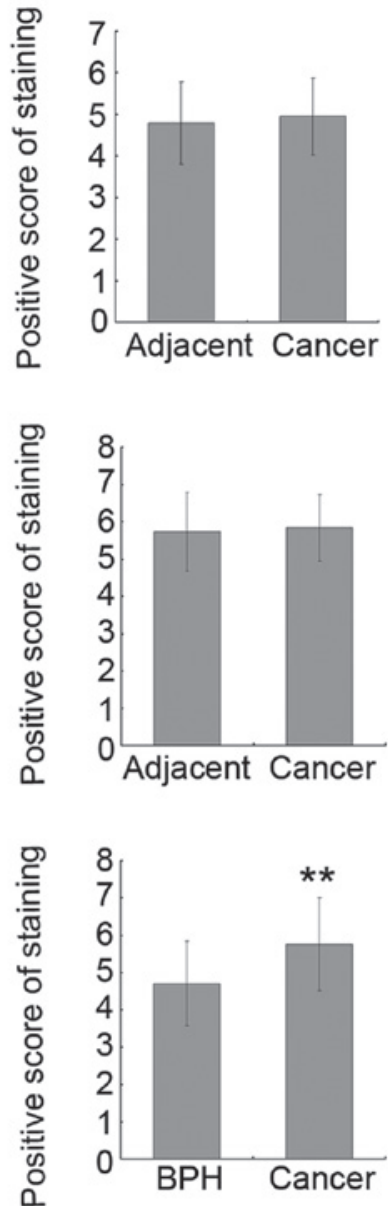

Figure 1. The global O-GlcNAc level is increased in prostate, but not in liver and pancreatic cancer tissues. Representative (A) liver, (B) pancreatic and (C) prostate cancer tissues incubated with the antibody targeting O-GlcNAc (RL2). Overall, 31 hepatocellular carcinoma and corresponding adjacent tissues, 31 pancreatic ductal adenocarcinoma and corresponding adjacent tissues, and 55 prostate cancer tissues [ 29 from prostate cancer tissue microarray (TMA) and 26 collected from the Qingdao Municipal Hospital (QMH)] and 48 benign prostatic hyperplasia (BPH) tissues (29 from the TMA and 19 collected from the $\mathrm{QMH}$ ) were assayed by immunoblotting. The staining intensity was graded by an experienced pathologist and analyzed by the Fromowitz standard. ${ }^{* *} \mathrm{P}<0.01$ (Student's t-test). Bars denote SD of the mean.

\section{Results}

The O-GlcNAc is increased in prostate cancer tissues. To investigate whether increased O-GlcNAcylation is universal in cancer, TMAs were used to detect the O-GlcNAc level in 31 hepatocellular carcinoma tissues (liver cancer), 31 pancreatic ductal adenocarcinoma tissues (pancreatic cancer), 29 prostate cancer tissues and their corresponding adjacent BPH tissues by immunohistochemistry, using an antibody targeting O-GlcNAc (RL2). Representative stained tissue sections are shown in the left and middle panels of Fig. 1. The intensity of O-GlcNAc immunostaining was analyzed by the Fromowitz standard. The results indicated that O-GlcNAcylation is increased in prostate cancer tissues as compared to the adjacent tissues; however, there was no significant difference between the liver/pancreatic cancer tissues and their corresponding adjacent tissues. To confirm the results observed in the prostate cancer tissues, 26 additional prostate cancer tissues and $19 \mathrm{BPH}$ tissues were collected and assayed for O-GlcNAc by immunohistochemistry. In accordance with the results from the TMA samples, the level of O-GlcNAc was increased in prostate cancer tissues as compared to BPH tissues, and statistical analysis on the combined dataset from TMAs and collected tissues showed that this increase is significant (Fig. 1C, right panel). These results suggested that O-GlcNAc may be a good molecular marker of prostate cancer and may play important roles in prostate cancer progression.

O-GlcNAc and OGT levels are increased in prostate cancer cells. To investigate whether O-GlcNAcylation is increased in all types of prostate cancer compared to BPH cells, we detected the global O-GlcNAc level in PC3, DU145 and BPH-1 cells by IB using the CTD110.6 antibody. Comparison of the global protein expression patterns in BPH-1, PC3 and DU145 cells by SDS-PAGE indicated that there is no obvious difference between these three cell lines (Fig. 2A). However, as shown in Fig. 2B and C, where immunoblots were treated and analyzed by ECL, the global O-GlcNAc level was significantly increased in the PC3 and DU145 compared to the BPH-1 cells, especially the staining intensity of the high molecular weight bands (marked with arrows, Fig. 2B); however, the intensity of the lower molecular weight bands (marked with stars, Fig. 2B) was weaker in the prostate cancer cell lines compared to the BPH-1 line.

Although there is only one OGT-encoding gene in mammalian genomes, alternative promoter usage and/or 
A

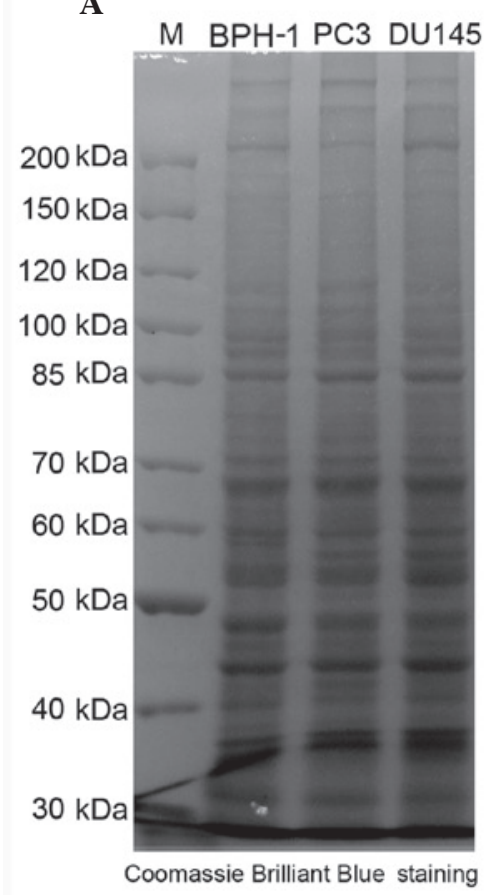

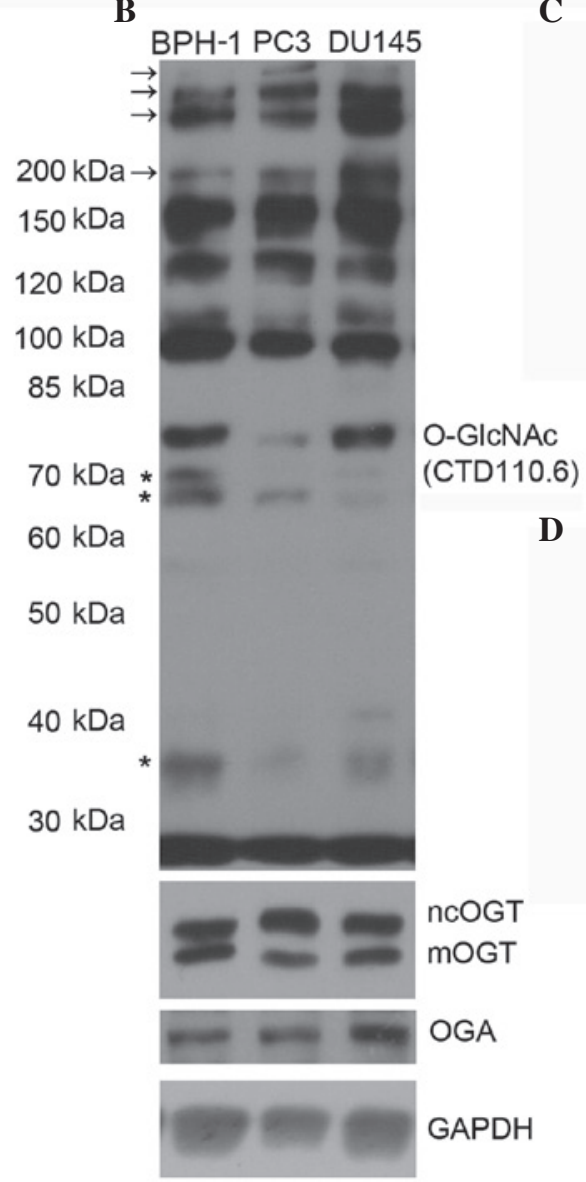

$\mathbf{C}$
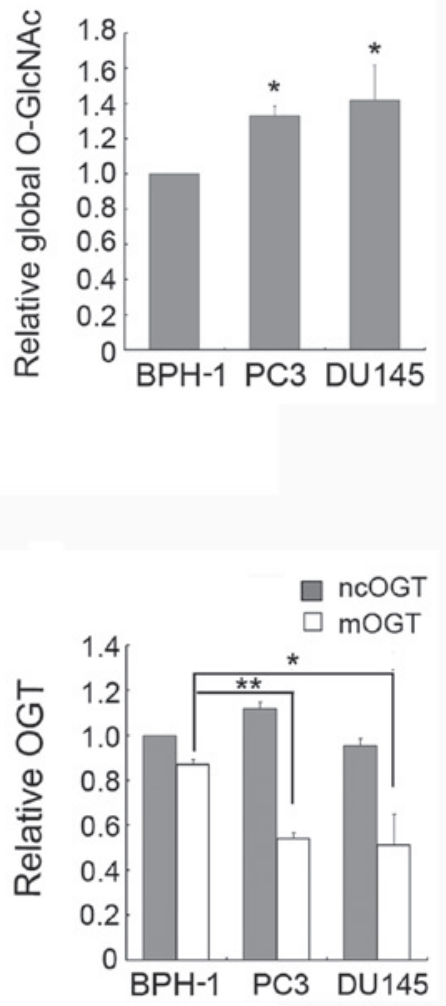

Figure 2. O-GlcNAc and OGT are detected in prostate cancer cells. (A) Whole-cell lysates of BPH-1, PC3 and DU145 were analyzed by $7.5 \%$ dodecyl sulfate-polyacrylamide gel electrophoresis (SDS-PAGE) and the gel was stained with Coomassie Brilliant Blue. M, marker. (B) The global O-GlcNAc, OGT, OGA and GAPDH levels were examined by immunoblotting using the anti-CTD110.6, -nucleocytoplasmic (nc) O-GlcNAc transferase (OGT), -mitochondrial (m)OGT, -O-GlcNAcase (OGA) and -glyceraldehyde 3-phosphate dehydrogenase (GAPDH) antibodies, respectively. The blots were visualized with enhanced chemiluminescence. Arrows $(\rightarrow)$ indicate high molecular weight bands with stronger staining intensity in prostate cancer cells, and stars (*) indicate low molecular weight bands with weaker staining intensity in prostate cancer cells. Quantification of the (C) global O-GlcNAc level and (D) ncOGT and mOGT levels following normalization with the GAPDH level. Bars denote $\mathrm{SD}$ of the mean from triplicate assays. ${ }^{*} \mathrm{P}<0.05 ;{ }^{* *} \mathrm{P}<0.01$ (Student's t-test).

alternative splicing produce three OGT isoforms, which differ in the length of their amino-terminal tetratricopeptide repeats (TPRs) (21). The nucleocytoplasmic OGT (ncOGT) contains 13 TPRs, the mitochondrial OGT (mOGT) contains a mitochondrial-targeting sequence at its $\mathrm{N}$-terminus and nine TPRs, and the shortest OGT isoform (sOGT) contains only two TPRs. Previous studies have indicated that the expression of OGT is increased in certain cancer types, but the OGT isoforms were not discriminated in these reports $(11,12,14,22)$. Here, we detected by IB the expression of OGT using specific antibodies that recognize all three OGT isoforms, and the results showed that ncOGT and mOGT, but not sOGT, are detectable in all tested cell lines (Fig. 2B). The expression of ncOGT was not significantly different between the prostate cancer and BPH cell lines, but the expression of mOGT was markedly decreased in prostate cancer cells (Fig. 2B and D). To our knowledge, this is the first report on decreased expression of the pro-apoptotic protein mOGT $(21,23)$ in cancer cells.

We also examined the expression of OGA in PC3, DU145 and BPH-1 cells, and the results indicated that the expression of OGA is increased in DU145 cells (Fig. 2B). Considering that the level of O-GlcNAc in DU145 cells was found increased, the

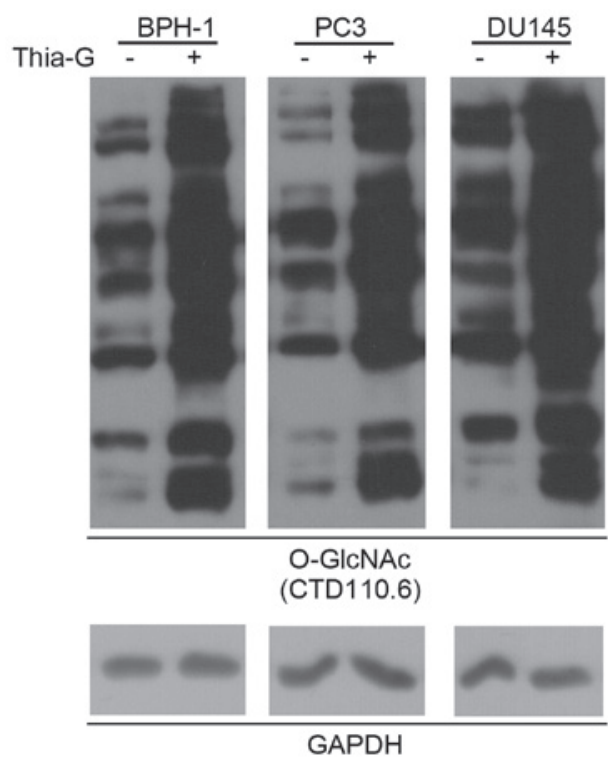

Figure 3. The O-GlcNAc level is increased upon thiamet-G (thia-G) treatment. BPH-1, DU145 and PC3 cells were pre-treated with $10 \mu \mathrm{M}$ thiamet-G for $24 \mathrm{~h}$ and subjected to immunoblotting using the CTD110.6 antibody targeting O-GlcNAc. Glyceraldehyde 3-phosphate dehydrogenase (GAPDH) was used as the loading control. 
increased expression of OGA may be due to feedback regulation.

O-GlcNAc enhances the anchorage-independent growth of BPH-1 and prostate cancer cells. To determine whether $\mathrm{O}-\mathrm{GlcNAc}$ is involved in prostate cancer malignancy, a highly potent and selective O-GlcNAcase inhibitor (thiamet-G) was used to increase the O-GlcNAc level in BPH-1, PC3 and DU145 cells (Fig. 3).

To investigate whether O-GlcNAc affects the proliferation and anchorage-independent growth of prostate cancer and BPH-1 cells, soft agar colony assays were performed. Thiamet-G treatment did not significantly affect the proliferation of the cells (MTT assay, data not shown). Soft agar colony assays showed that in the presence of thiamet-G, BPH-1, DU145 and PC3 cells form colonies at days 5-7 (Fig. 4); however, without thiamet-G, DU145 and PC3 cells were able to form colonies only two weeks later, while BPH-1 cells were not able to form colonies (data not shown).

Thiamet- $\mathrm{G}$ treatment not only enhanced colony formation in the prostate cancer cell lines DU145 and PC3, but further endowed BPH-1 cells with the ability to form colonies. These results indicated that O-GlcNAc is important for the anchorage-independent growth of prostate cancer cells, suggesting that increased O-GlcNAcylation may be sufficient for the malignant transformation of BPH cells.

O-GlcNAc enhances the invasion of prostate cancer cells. To investigate whether O-GlcNAc affects the invasive ability of prostate cancer cells, invasion assays were performed. As shown in Fig. 5, the invasive ability of DU145 (Fig. 5B) and PC3 cells (Fig. 5C) was significantly enhanced by thiamet-G treatment. However, neither BPH-1 nor the thiamet-G-treated BPH-1 cells showed invasive ability in our assays (data not shown); we thus next investigated the role of O-GlcNAc in BPH-1 migration. The results showed that O-GlcNAc markedly enhanced migration in BPH-1 cells (Fig. 5A). Overall, these results suggested that O-GlcNAcylation may play important roles in prostate cancer metastasis.

O-GlcNAc inhibits the formation of the E-cadherin/catenin complex. E-cadherin-mediated cel-cell adhesion is generally reduced in human cancers, and loss of E-cadherin is associated with tumor invasiveness, metastatic dissemination, and poor patient prognosis (24). In addition, Snaill was reported to be stabilized by O-GlcNAcylation and thus, inhibit the transcription of E-cadherin (25). We therefore investigated whether O-GlcNAc can affect the expression of E-cadherin in prostate cancer cells. BPH-1, PC3 and DU145 cells were treated with thiamet-G for $24 \mathrm{~h}$ and the mRNA level of $E$-cadherin was detected by quantitative RT-PCR. The results indicated that the elevation of O-GlcNAc does not affect the transcription of $E$-cadherin in any of the three cell lines (Fig. 6A). The mRNA level of E-cadherin was lower in DU145 and PC3 compared to BPH-1 cells.

We previously reported that increased O-GlcNAcylation reduces the level of the cell surface protein E-cadherin, which has the potential to enhance cell invasion and metastasis in breast cancer cells (12). The binding of the E-cadherin/catenin complex to the cytoskeleton is essential
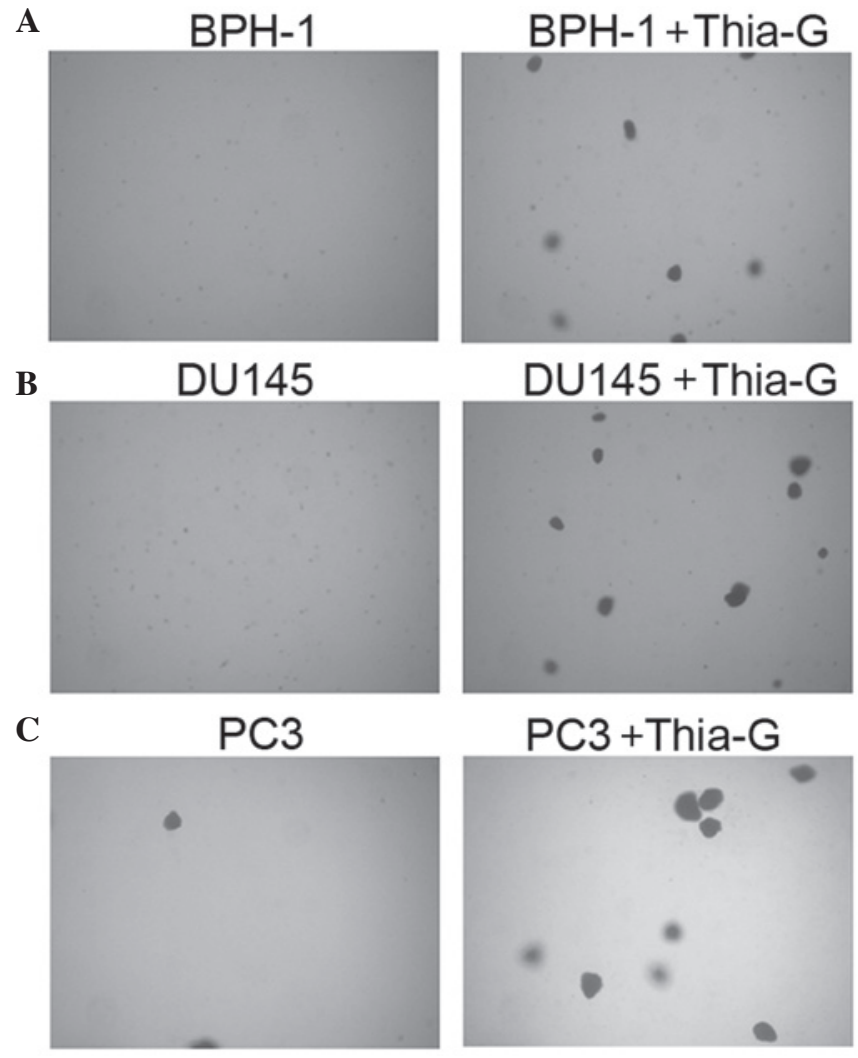

Figure 4. O-GlcNAc enhances anchorage-independent growth of BPH-1, DU145 and PC3 cells. The anchorage-independent growth of (A) BPH-1, (B) DU145 and (C) PC3 cells was analyzed by the soft agar assay with or without $10 \mu \mathrm{M}$ thiamet-G (thia-G). On the 5th day, images were acquired from 6 random fields of the colonies at $x 40$ magnification.

for strong cell-cell adhesion (26), and we demonstrated that an increase in the O-GlcNAc level inhibits the formation of the E-cadherin/catenin/cytoskeleton complex (12). Here, the E-cadherin/catenin/cytoskeleton complex was isolated from BPH-1 and DU145 cell extracts in the Triton X-100-insoluble fraction. The levels of the cytoskeleton-associated proteins E-cadherin and p120 were reduced in the thiamet-G-treated BPH-1 (Fig. 6B and C) and DU145 (Fig. 6B and D) cells, suggesting that the reduction in the level of the cell surface protein E-cadherin may cause the enhanced migratory and invasive ability of prostate cancer cells.

\section{Discussion}

In previous studies, we analyzed histological sections from breast, lung and colon tumors and demonstrated that the $\mathrm{O}-\mathrm{GlcNAc}$ level is increased in these tissues compared to matched adjacent tissues $(11,12)$. Similarly, in patients with chronic lymphocytic leukaemia, the O-GlcNAc level was increased when compared to healthy lymphocytes (27). Similar results were also obtained in breast (14), prostate (13) and pancreatic cancer cell lines (15) by IB analysis. A most likely reason for the elevation of O-GlcNAcylation in cancer tissues/cells is the increased rate of glucose utilization, which may enhance the HBP pathway, and thus increase the concentration of UDP-GlcNAc in cancer cells. In line with this hypothesis, it has been reported that the concentration of UDP-GlcNAc is increased in chronic lymphocytic leukemia 
A

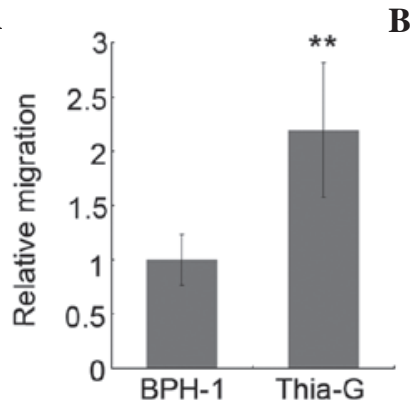

B

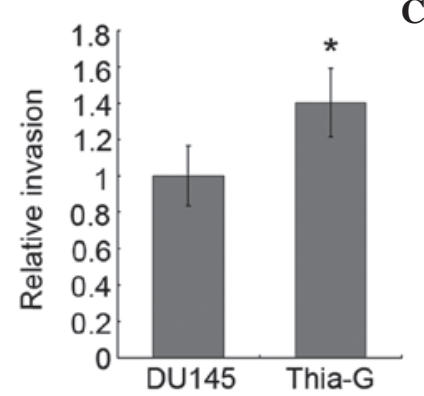

C

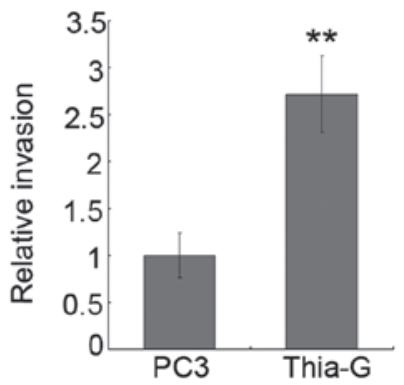

Figure 5. Increased O-GlcNAcylation reduces the migratory/invasive ability of BPH-1, DU145 and PC3 cells. Thiamet-G (thia-G) treatment significantly enhances (A) migration in BPH-1 cells and invasion in (B) DU145 and C) PC 3 cells. Bars denote SD of the mean from triplicate repetitions. ${ }^{* *} \mathrm{P}<0.01,{ }^{*} \mathrm{P}<0.05$ (Student's t-test).

A

B
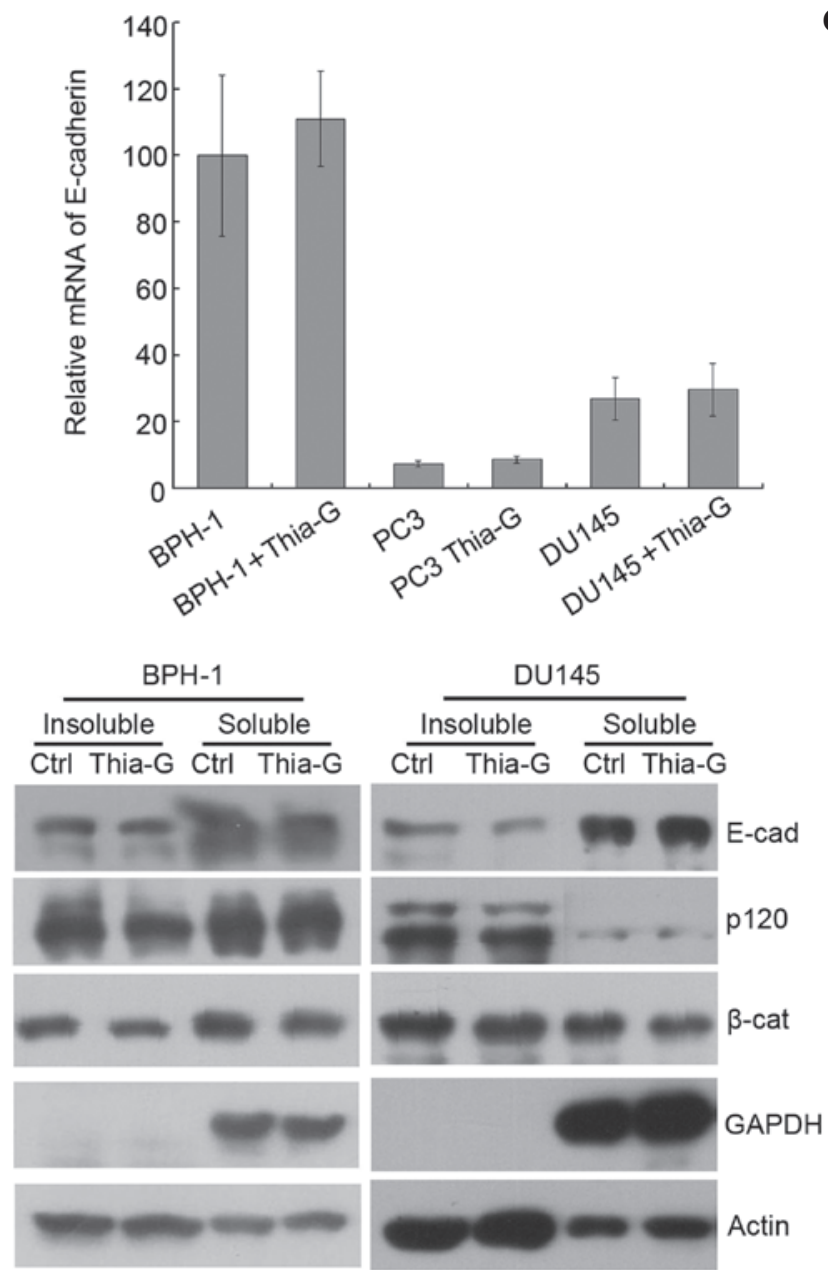

C

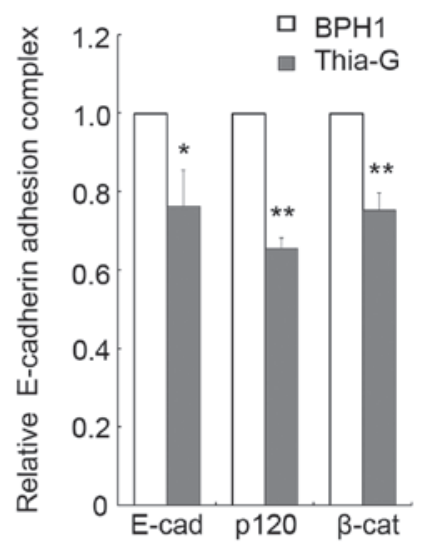

D

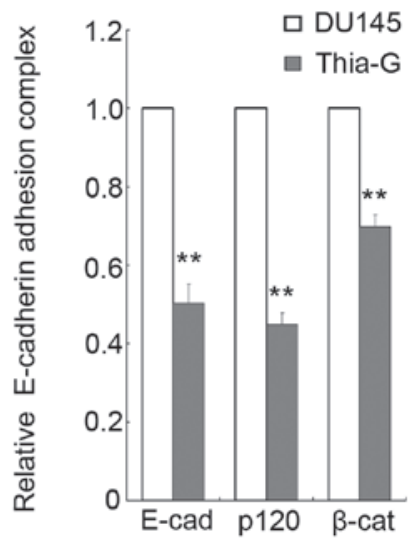

Figure 6. O-GlcNAc inhibits the formation of the E-cadherin/catenin/cytoskeleton complex. BPH-1, DU145 and PC3 cells were pretreated with or without $10 \mu \mathrm{M}$ thiamet-G (thia-G) for $24 \mathrm{~h}$. (A) Quantitative reverse transcription (RT)-PCR was used to detect the relative mRNA level of E-cadherin. (B) BPH-1 and DU145 cells were lysed in Triton X-100 lysis buffer to obtain cytoskeleton-binding proteins (Triton X-100-insoluble fraction) and proteins that do not bind to the cytoskeleton (Triton X-100-soluble fraction), and then immunoblotted with antibodies targeting E-cadherin (E-cad), p120, $\beta$-catenin ( $\beta$--cat), $\beta$-actin and glyceraldehyde-3-phosphate dehydrogenase (GAPDH). Quantification of the cytoskeleton-binding E-cadherin, p120 and $\beta$-catenin levels following normalization with the level of GAPDH in (C) BPH-1 and (D) DU145 cells. Bars denote SD of the mean from triplicate repetitions. ${ }^{* * *} \mathrm{P}<0.01$, "P<0.05 (Student's t-test).

cells (27). Based on all the above, we argue that the increase in O-GlcNAc may be a universal event in different types of cancer that show alterations in glucose metabolic pathways (Warburg effect). However, we found that the levels of O-GlcNAc are not increased in liver and pancreatic cancer tissues. Two explanations for this contradictory result may be postulated: first, there is no report on the systematic comparison of the concentration of UDP-GlcNAc in solid cancer tissues or cells and their healthy counterparts. Therefore, we can not confirm whether UDP-GlcNAc is increased in different types of cancer. Second, a previous study reported that the apparent $\mathrm{Km}$ (Kmapp) values of OGT 
for UDP-GlcNAc vary depending on the protein substrate, suggesting that certain OGT substrates are nutrient-responsive, whereas others are constitutively modified (28). The constitutively modified proteins are more easily modified by $\mathrm{O}-\mathrm{GlcNAc}$, and thus have a higher O-GlcNAc stoichiometry; these proteins may be the main contributors of the increased global O-GlcNAc level observed by immunohistochemistry in cancer tissues/cells. In conclusion, the increase in global O-GlcNAcylation may not be a suitable marker for cancer; it may be more relevant to consider O-GlcNAcylation in specific proteins.

In contrast to our results, a recent report indicated that O-GlcNAcylation is globally increased in pancreatic ductal adenocarcinoma cell lines compared to non-transformed human pancreatic duct epithelial cells. In addition, the study also reported that pancreatic cancer tissue exhibits increased O-GlcNAc staining compared to healthy pancreatic ducts (15). However, this study was restricted to a limited number and type of cells and only used one tissue specimen. A potential explanation for the different results with our study may be the screening of different tissues.

Zhu et al compared the O-GlcNAc levels of forty hepatocellular carcinoma (HCC) tissues to ten healthy liver tissues by immunohistochemistry analysis. The authors found that global O-GlcNAcylation is significantly increased in HCC tissues compared to healthy liver tissues (29). By contrast, our study did not find a significant difference in the global O-GlcNAc level between hepatocellular carcinoma tissues and their matched adjacent tissues. One reason for the contradiction may be the fact that liver cancer adjacent tissues used in our study were chronic hepatitis, but not healthy liver tissues. Liver tissue inflammation may cause the deregulation in O-GlcNAcylation.

In this study, we found that global O-GlcNAc levels are increased in prostate cancer tissues compared to the BPH tissues, which was consistent with our previous finding that $\mathrm{O}-\mathrm{GlcNAc}$ levels are increased in prostate cancer cell lines compared to the healthy prostate epithelium. As discussed above, the increase in the UDP-GlcNAc level may be the main cause for the upregulation of global O-GlcNAcylation in prostate cancer. However, another study showed that the increased expression of OGT may be an additionally important positive regulator of O-GlcNAcylation in prostate cancer (22). In this study, we detected the global O-GlcNAc and OGT levels in BPH-1, PC3 and DU145 cells. Although the global O-GlcNAc level was slightly increased in prostate cancer cells, the O-GlcNAc staining intensities were not homogeneously increased in all bands: the intensities of some O-GlcNAc-stained bands from prostate cancer cells were increased, while the intensities of other bands were decreased compared to BPH-1 cells. This might be explained by the different target protein preference of OGT in prostate cancer and BPH-1 cells.

Our study also demonstrated that O-GlcNAc can enhance the malignancy of several cancer types, and that the OGT level is increased in certain cancer cell lines. However, we found that the expression of mOGT is decreased in prostate cancer cells compared to BPH-1 cells. Considering that mOGT has pro-apoptotic roles, the reduced expression of mOGT may enhance the survival and anti-apoptotic abilities of prostate cancer cells. The roles of mOGT in cancer formation and progression need to be further investigated.

The human prostatic epithelial cell line BPH-1, derived from BPH tissue and immortalized with the SV40 large T antigen, is normally nontumorigenic in nude mice (30) and can not form colonies in the soft agar transformation assay. BPH-1 can be induced to form tumors by various factors, such as association with a tumorigenic stromal microenvironment, treatment with hormonal carcinogens or by oncogene transformation $(31,32)$. In this study, we found that the increase in O-GlcNAcylation by thiamet-G induced anchorage-independent growth and migration in BPH-1 cells, which is the first direct evidence that O-GlcNAc can induce malignant transformation in bening cells. It is notable that thiamet-G treatment induced colony formation in BPH-1, PC3 and DU145 cells on the 4th-7th day during the soft agar assay, while two weeks were required for colony formation in the non-treated PC3 and DU145 cells (data not shown). Moreover, we found that thiamet-G treatment enhances invasion in PC3 and DU145 cells. Consistent with our results, Lynch et al reported that the $O G T$ knock-down in prostate cancer cells inhibits anchorage-independent cell growth, cell invasion and angiogenesis (13). Based on these results, we propose that O-GlcNAc is involved in all steps of prostate cancer formation and progression.

E-cadherin is a well-known cell-cell adhesion molecule, which acts as a suppressor of cancer cell invasion; its expression is downregulated in numerous types of advanced human cancer. There have been some reports that provided evidence on the regulation of E-cadherin by O-GlcNAc. For example, O-GlcNAc inhibited the transcription of E-cadherin by modifying and stabilizing Snail1 (25); our group also reported that the increase in the O-GlcNAc level can inhibit the formation of the E-cadherin/catenin/cytoskeleton complex, and thus induce invasion and metastasis in breast cancer cells (12). In the present study, we found that the increase in the O-GlcNAc level does not affect the mRNA level of E-cadherin in BPH-1, PC3 and DU145 cells, but that O-GlcNAc inhibited the formation of the E-cadherin/catenin/cytoskeleton complex. Therefore, regulation of E-cadherin might constitute the mechanism underlying the induction of prostate cancer invasion. However, the invasive ability of PC 3 cells, in which E-cadherin is expressed at low levels, was also significantly enhanced in our study. Furthermore, it was reported that reducing the expression of OGT leads to increased degradation of the FoxM1 protein, and thus regulates the progression of breast and prostate cancer cells (13). Moreover, there are numerous proteins related to tumor progression that are modified and regulated by O-GlcNAc (8). Overall, previous reports and present findings suggest that the mechanisms involved in the roles of O-GlcNAc in cancer progression may be multidimensional and/or highly context-dependent.

In summary, we showed that global O-GlcNAcylation is increased in prostate, but not liver and pancreatic cancer tissues compared to their corresponding adjacent tissues, and that the expression of mOGT, but not ncOGT, is decreased in prostate cancer cell lines; moreover, we found that $\mathrm{O}-\mathrm{GlcNAc}$ can enhance the malignancy of prostate cancer cells, at least partially through inhibition of the formation of the E-cadherin/catenin/cytoskeleton complex. 


\section{Acknowledgements}

We would like to thank Dr Xinzhi Lu, Feng Han and Qianhong Gong for numerous discussions. This work was supported by the National Natural Science Foundation of China (grant nos. 81101505, 81172013 and 81272264) and the Open Research Fund Program of the Key Laboratory of Marine Drugs [grant no. KLMD(OUC)201308].

\section{References}

1. Banerjee PS, Hart GW and Cho JW: Chemical approaches to study O-GlcNAcylation. Chem Soc Rev 42: 4345-4357, 2013.

2. Marshall S, Bacote V and Traxinger RR: Discovery of a metabolic pathway mediating glucose-induced desensitization of the glucose transport system. Role of hexosamine biosynthesis in the induction of insulin resistance. J Biol Chem 266: 4706-4712, 1991.

3. Kreppel LK and Hart GW: Regulation of a cytosolic and nuclear O-GlcNAc transferase. Role of the tetratricopeptide repeats. J Biol Chem 274: 32015-32022, 1999.

4. Park SY, Ryu J and Lee W: O-GlcNAc modification on IRS-1 and Akt 2 by PUGNAc inhibits their phosphorylation and induces insulin resistance in rat primary adipocytes. Exp Mol Med 37: 220-229, 2005.

5. Yang X, Ongusaha PP, Miles PD, et al: Phosphoinositide signalling links O-GlcNAc transferase to insulin resistance. Nature 451: 964-969, 2008.

6. Buse MG: Hexosamines, insulin resistance, and the complications of diabetes: current status. Am J Physiol Endocrinol Metab 290: E1-E8, 2006.

7. Liu F, Iqbal K, Grundke-Iqbal I, Hart GW and Gong CX: O-GlcNAcylation regulates phosphorylation of tau: a mechanism involved in Alzheimer's disease. Proc Natl Acad Sci USA 101 10804-10809, 2004.

8. Slawson C and Hart GW: O-GlcNAc signalling: implications for cancer cell biology. Nat Rev Cancer 11: 678-684, 2011.

9. Warburg O: On the origin of cancer cells. Science 123: 309-314, 1956.

10. Pecqueur C, Oliver L, Oizel K, Lalier L and Vallette FM: Targeting metabolism to induce cell death in cancer cells and cancer stem cells. Int J Cell Biol 2013: 805975, 2013.

11. Mi W, Gu Y, Han C, et al: O-GlcNAcylation is a novel regulator of lung and colon cancer malignancy. Biochim Biophys Acta 1812 514-519, 2011.

12. Gu Y, Mi W, Ge Y, et al: GlcNAcylation plays an essential role in breast cancer metastasis. Cancer Res 70: 6344-6351, 2010.

13. Lynch TP, Ferrer CM, Jackson SR, Shahriari KS, Vosseller K and Reginato MJ: Critical role of O-linked $\beta$-N-acetylglucosamine transferase in prostate cancer invasion, angiogenesis, and metastasis. J Biol Chem 287: 11070-11081, 2012.

14. Caldwell SA, Jackson SR, Shahriari KS, et al: Nutrient sensor $\mathrm{O}-\mathrm{GlcNAc}$ transferase regulates breast cancer tumorigenesis through targeting of the oncogenic transcription factor FoxM1 Oncogene 29: 2831-2842, 2010 .
15. Ma Z, Vocadlo DJ and Vosseller K: Hyper-O-GlcNAcylation is anti-apoptotic and maintains constitutive NF- $\kappa \mathrm{B}$ activity in pancreatic cancer cells. J Biol Chem 288: 15121-15130, 2013.

16. Fromowitz FB, Viola MV, Chao S, et al: ras p21 expression in the progression of breast cancer. Hum Pathol 18: 1268-1275, 1987.

17. Qin JM, Fu XY, Li SJ, et al: Gene and protein expressions of p28GANK in rat with liver regeneration. World J Gastroenterol 9: 2523-2527, 2003.

18. Yuzwa SA, Macauley MS, Heinonen JE, et al: A potent mechanism-inspired O-GlcNAcase inhibitor that blocks phosphorylation of tau in vivo. Nat Chem Biol 4: 483-490, 2008.

19. Gu Y, Zhang J, Mi W, et al: Silencing of GM3 synthase suppresses lung metastasis of murine breast cancer cells. Breast Cancer Res 10: R1, 2008

20. Zhu W, Leber B and Andrews DW: Cytoplasmic O-glycosylation prevents cell surface transport of E-cadherin during apoptosis. Embo J 20: 5999-6007, 2001.

21. Love DC, Kochan J, Cathey RL, Shin SH and Hanover JA: Mitochondrial and nucleocytoplasmic targeting of O-linked GlcNAc transferase. J Cell Sci 116: 647-654, 2003.

22. Itkonen HM, Minner S, Guldvik IJ, et al: O-GlcNAc transferase integrates metabolic pathways to regulate the stability of c-MYC in human prostate cancer cells. Cancer Res 73: 5277-5287, 2013.

23. Shin SH, Love DC and Hanover JA: Elevated O-GlcNAc-dependent signaling through inducible mOGT expression selectively triggers apoptosis. Amino Acids 40: 885-893, 2011

24. Hirohashi S: Inactivation of the E-cadherin-mediated cell adhesion system in human cancers. Am J Pathol 153: 333-339, 1998.

25. Park SY, Kim HS, Kim NH, et al: Snaill is stabilized by O-GlcNAc modification in hyperglycaemic condition. EMBO J 29: 3787-3796, 2010.

26. Pokutta $S$ and Weis WI: Structure and mechanism of cadherins and catenins in cell-cell contacts. Annu Rev Cell Dev Biol 23: 237-261, 2007.

27. Shi Y, Tomic J, Wen F, et al: Aberrant O-GlcNAcylation characterizes chronic lymphocytic leukemia. Leukemia 24: 1588-1598, 2010

28. Shen DL, Gloster TM, Yuzwa SA and Vocadlo DJ: Insights into O-linked N-acetylglucosamine ([0-9]O-GlcNAc) processing and dynamics through kinetic analysis of O-GlcNAc transferase and O-GlcNAcase activity on protein substrates. J Biol Chem 287: 15395-15408, 2012.

29. Zhu Q, Zhou L, Yang Z, et al: O-GlcNAcylation plays a role in tumor recurrence of hepatocellular carcinoma following liver transplantation. Med Oncol 29: 985-993, 2012.

30. Hayward SW, Dahiya R, Cunha GR, Bartek J, Deshpande N and Narayan P: Establishment and characterization of an immortalized but non-transformed human prostate epithelial cell line: BPH-1. In Vitro Cell Dev Biol Anim 31: 14-24, 1995.

31. Hayward SW, Wang Y, Cao M, et al: Malignant transformation in a nontumorigenic human prostatic epithelial cell line. Cancer Res 61: 8135-8142, 2001.

32. Webber MM, Quader ST, Kleinman HK, et al: Human cell lines as an in vitro/in vivo model for prostate carcinogenesis and progression. Prostate 47: 1-13, 2001. 